\title{
Kinetic factors determining wheelchair propulsion in marathon racers with paraplegia
}

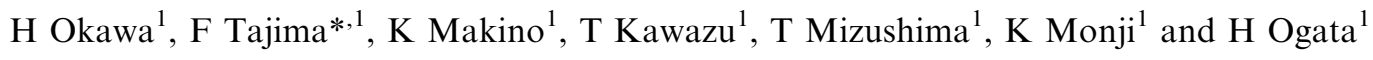 \\ ${ }^{1}$ Department of Rehabilitation Medicine, University of Occupational and Environmental Health, Japan, Fukuoka 807- \\ 8555, Japan
}

Objective: To assess the different factors that determine the propulsion of wheelchair in long distance racers with spinal cord injuries.

Design and methods: Nine highly handicapped men who competed at the Oita International Wheelchair Marathon in 1995, participated in this study. Subjects performed steady and exhaustive wheelchair propulsion at their optimal speed on wheelchair rollers in a laboratory setting during a 5-min period. Movements of the trunk and arms were filmed by a video camera then analyzed kinetically using a two-dimensional analysis system. In order to determine the actual pushing time and angle of wheelchair, we measured the duration between hand contact and hand release from handrim.

Setting: Department of Rehabilitation Medicine University of Occupational and Environmental Health, Japan.

Results: The push time, cycle time, total push time, and angular velocity did not relate with the race time or wheelchair velocity. However, the total push angle correlated significantly $(P<0.01)$ with the race time and wheelchair velocity. Improvement of the pushing angular velocity and pushing duration did not contribute to the race time.

Conclusion: The skilful wheelchair propulsion in long distance racing depends on the total push angle. Improvement of the total push angle should be achieved by both prolongation of the total push time and increased pushing angular velocity.

Keywords: arm movements; spinal cord injuries; wheelchair marathon

\section{Introduction}

Wheelchair sports for persons with disabilities have greatly improved the physical fitness and social interaction of these individuals. Recently, wheelchair sports competition events have become popular and numerous athletes with disabilities continue to train and compete. Wheelchair marathon racing is one of the best organized and most symbolic wheelchair sports event, and there are numerous international wheelchair marathons. ${ }^{1}$ While some physiological factors might influence actual race time, such as cardiorespiratory endurance, muscular strength, and motivational level, there are no reports regarding the relationship between the kinetic skill of wheelchair propulsion and the race times. Therefore, there is a general concern about the training strategies used to improve the performance of wheelchair long distance racers. Furthermore, very few coaches or athletes understand the technical aspects of wheelchair

*Correspondence: F Tajima, MD, PhD, Assistant Professor, Department of Rehabilitation Medicine, University of Occupational and Environmental Health, Japan, 1-1 Iseigaoka Yahatanishi-ku, Kitakyushu-shi, Fukuoka-ken 807-8555, Japan propulsion which may improve performance and shorten the race time.

Instruction and basic studies have examined the technical aspects of wheelchair propulsion. ${ }^{2-5}$ The cycle pattern in wheelchair propulsion can be divided into two phases that together form one complete cycle: (a) the push or contact phase, during which the hands are in contact with the rim and exert force with time, and (b) the recovery phase during which the arms are released from the rim and brought back to a position to begin a new propulsive act. The stroke frequency is defined as the number of cycles per unit time. ${ }^{5}$ Most studies focused on the efficiency of pushing the rim, while the arm movements have been observed at different velocities on a motor driven treadmill in the same subjects. To our knowledge, no study has been performed to provide long distance wheelchair racers with some guidelines for determining the optimal arm movement pattern that increases performance and perhaps to win the race.

Effective wheelchair propulsion depends on two strategies. One is using a fast velocity in pushing the handrim (angular velocity of pushing the hand during the pushing phase), which translates into a great acceleration during pushing the handrim. The 
other is prolongation of the actual propulsion time, since the long duration of the applied force transfers from the hands to the hand rims and increases acceleration, and hence the velocity of the wheelchair. The long pushing duration is achieved by either the use of frequent wheelchair propulsions or a prolonged handrim pushing time during one cycle (push time or prolongation of handrim pushing time during $1 \mathrm{~min}$, total push time). Finally, the total product of angular velocity and total push time (total push angle) might be used to achieve the best wheelchair propulsion in the wheelchair long distance race. We hypothesized that kinetically important technical factors of

Table 1 Anthropometric data and disability of the athletes participating in the study

\begin{tabular}{lcccc}
\hline Subject & $\begin{array}{c}\text { Age } \\
\text { (years })\end{array}$ & $\begin{array}{c}\text { Height } \\
(\mathrm{cm})\end{array}$ & $\begin{array}{c}\text { Weight } \\
(\mathrm{kg})\end{array}$ & $\begin{array}{c}\text { Disability } \\
\text { level }\end{array}$ \\
\hline A & 37 & 178 & 60 & $\mathrm{~T} 4$ \\
$\mathrm{~B}$ & 30 & 167 & 46 & $\mathrm{~L} 1$ \\
$\mathrm{C}$ & 34 & 171 & 60 & $\mathrm{~T} 12$ \\
$\mathrm{D}$ & 21 & 176 & 65 & $\mathrm{~T} 7$ \\
$\mathrm{E}$ & 24 & 180 & 70 & $\mathrm{~T} 12$ \\
$\mathrm{~F}$ & 20 & 175 & 65 & $\mathrm{~T} 12$ \\
$\mathrm{G}$ & 47 & 169 & 66 & $\mathrm{~T} 12$ \\
$\mathrm{H}$ & 46 & 171 & 48 & $\mathrm{~T} 4$ \\
$\mathrm{I}$ & 43 & 165 & 62 & $\mathrm{~T} 4$ \\
\hline
\end{tabular}

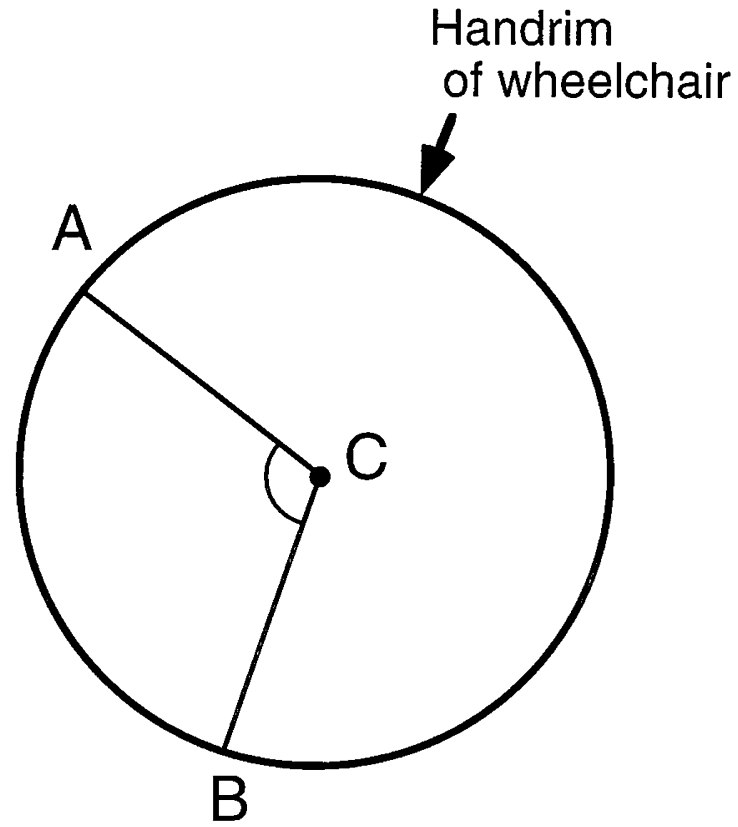

Figure 1 Illustration of the notion of the push time and push angle. A: the point of hand contact to handrim of wheelchair, B: the point of hand release from handrim of wheelchair, C: the center of the handrim of wheelchair. See the text for details

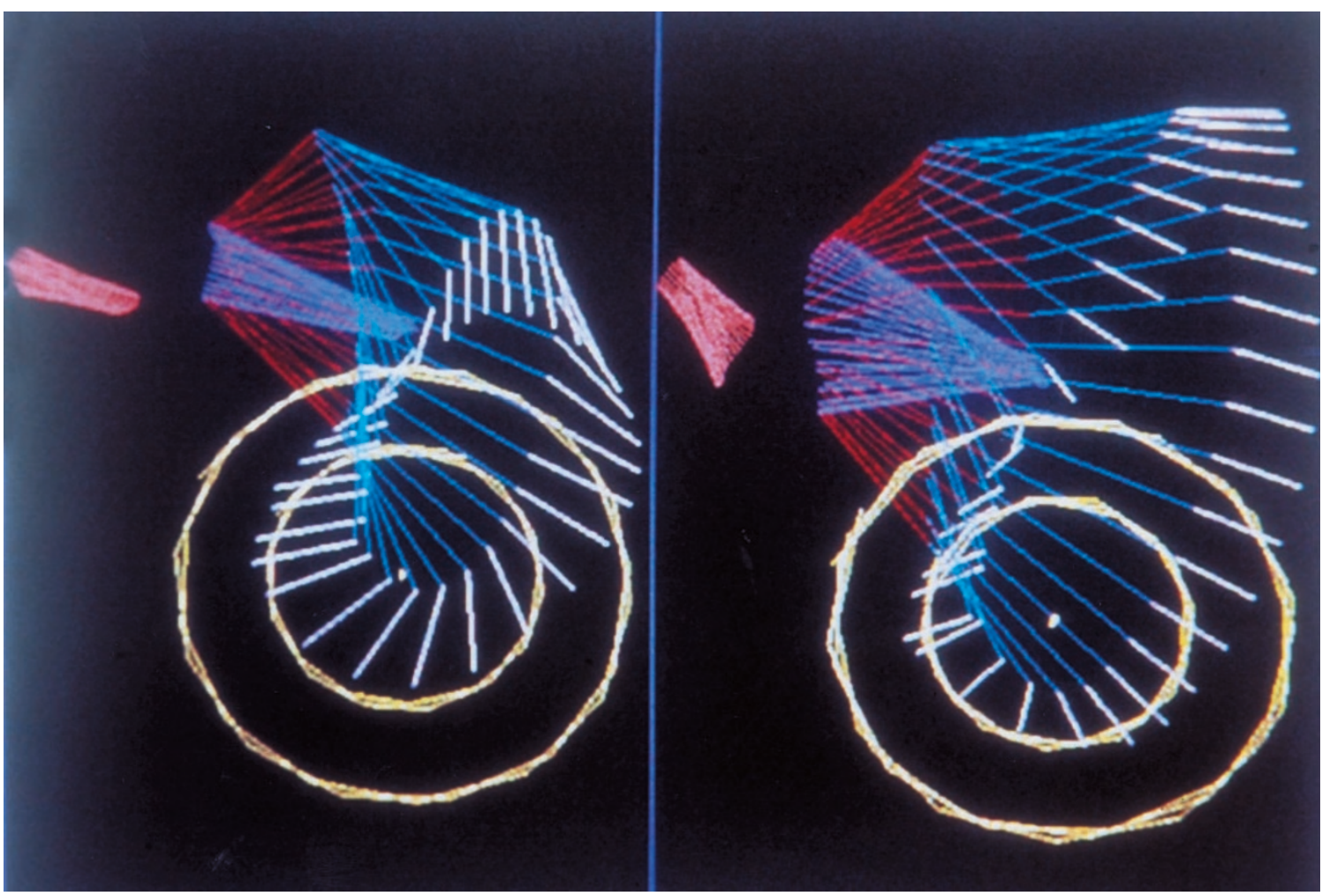

Figure 2 Typical results of kinematics analysis. See the text for details 
wheelchair propulsion in long distance wheelchair race include (1) angular velocity, (2) push time, (3) total push time, and (4) total push angle.

The purpose of this study was to examine the relationship between the kinetic factors and the race records of paraplegic marathon-racers who competed in the Oita International wheelchair marathon race in 1995. In the present study, the arm movement pattern at propelling a wheelchair on the roller in a laboratory

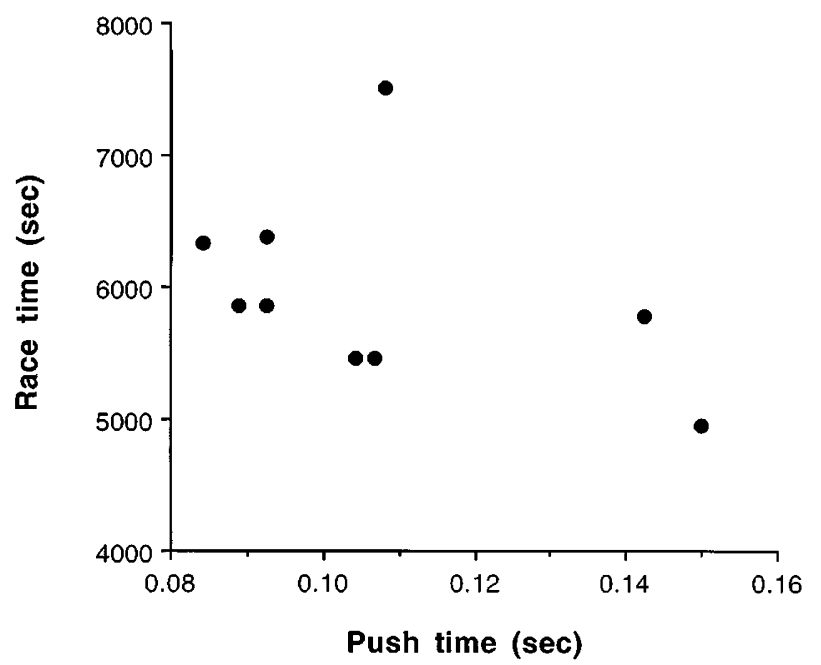

Figure 3 Relationship between push time and race time. Each data point represents the mean of eight consecutive strokes of each subject during the last minute of the 5-min propulsion

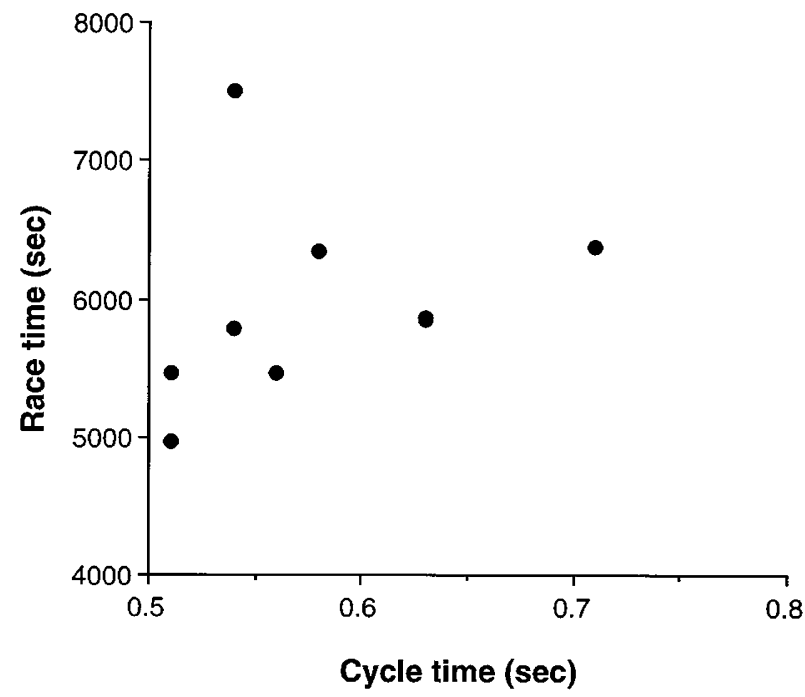

Figure 4 Relationship between cycle time and race time. Each data point represents the mean of eight consecutive strokes of each subject during the last minute of the 5-min propulsion setting was recorded and analzyed using two dimensional analysis system.

\section{Methods}

\section{Subjects}

Nine highly trained male subjects with spinal cord injuries participated in the present study. All subjects competed in the full-marathon division of the Oita International Wheelchair Marathon Race in 1995. Their anthropometric characteristics and disability level are shown in Table 1. A written informed consent was obtained from each subject.

\section{Test protocol}

Each subject was tested using his own wheelchair in a simulated long distance road race using a wheelchair roller (TOP END ACTION, CO, USA) in our laboratory. The subjects performed 5 min steady and exhaustive wheelchair propulsion as quickly as they could.

\section{Measurements}

The wheelchair propulsion style was recorded throughout the experiment, and movement patterns were filmed by a video camera. Markers were placed on the top of the head, ear, acromion, lateral epicondyle of the humerus, styloid process of the ulna, tip of the thumb, iliac crest, the rear wheel axis, handrim, and rear wheel. Movement patterns were analyzed using a microcomputer-based system (Peak Performance Technologies Inc., Englewood, CO, USA). The location of

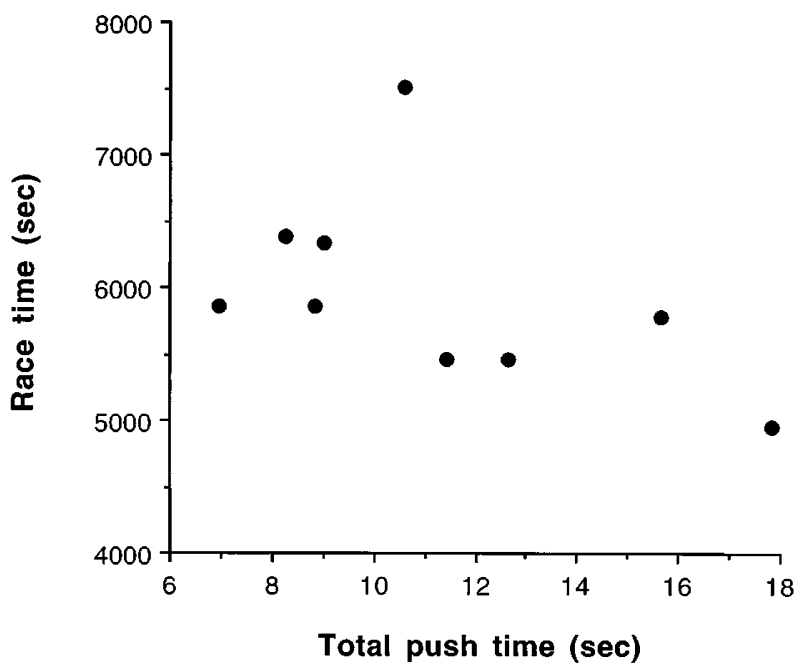

Figure 5 Relationship between total push time and race time. Each data point represents the mean of eight consecutive strokes of each subject during the last minute of the 5-min propulsion 




Figure 6 Relationship between angular velocity and race time. Each data point represents the mean of eight consecutive strokes of each subject during the last minute of the 5-min propulsion

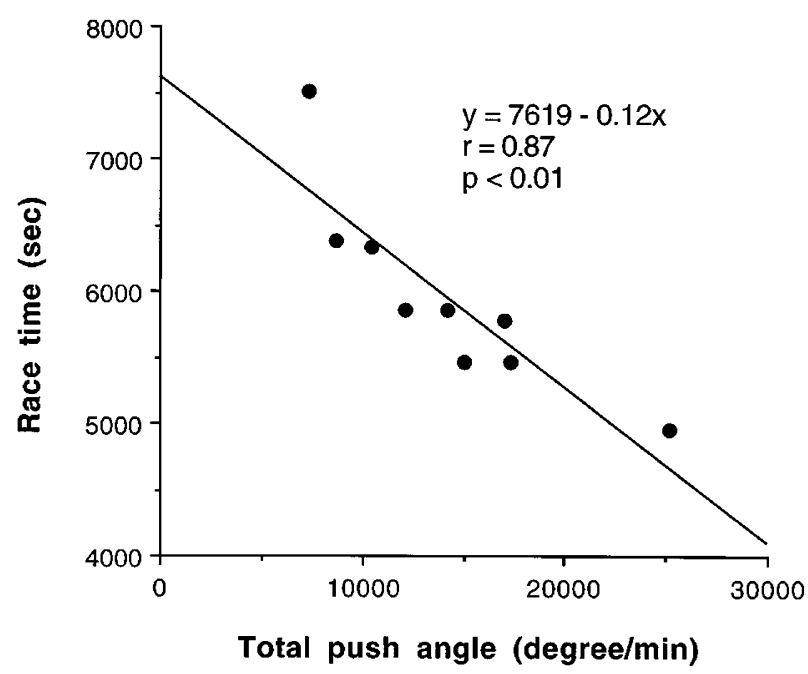

Figure 7 Relationship between total push angle and race time. Each data point represents the mean of eight consecutive strokes of each subject during the last minute of the 5-min propulsion

each marker was digitized at 60 frames/s and all kinetic data of eight consecutive strokes during the last minute of the $5 \mathrm{~min}$ test were collected. After digitization of the images, two-dimensional reconstruction was performed using the direct linear transformation method. When marker positions were concealed due to segmental rotation, their positions were estimated by linear interpolation.

After identifying the points of hand contact and hand release, the start angle and end angle of the push range were calculated relative to the horizontal through the rear axle and were defined as the vector from the rear axle to the marker on the tip of the thumb. The push angle was defined as the difference between start angle and end angle (the angle of A, C and $\mathrm{B}$ in Figure 1). Several time parameters, including the cycle time, push time (the time duration between hand contact to the point $\mathrm{A}$ and hand release from the point B in Figure 1), and recovery time, were derived based on the number of film frames. ${ }^{5}$ The cycle frequency represented the average number of wheel cycles per minute. The angular velocity (= push angle/ push time) and total push time (=push time $\times$ cycle frequency) were also calculated. The skilful push of the handrim, termed total push angle, represented a momentum of the angular velocity and duration of pushing. The total push angle was calculated using the following formula;

Total push angle $=$ angular velocity $\times$ total push time

$$
\begin{aligned}
= & {[(\text { push angle } / \text { push time }) \times} \\
& (\text { push time } \times \text { cycle frequency })] \\
= & \text { push angle } \times \text { cycle frequency. }
\end{aligned}
$$

Statistical analysis

The relationships between each of several parameter and race time at the Oita International Wheelchair Marathon Race in 1995 was analyzed using linear regression analysis. The level of significance was selected at $P<0.05$.

\section{Results}

Graphic analysis of the movement pattern showed the position of the upper limb at the beginning and end of the cycle was always exactly the same. This finding is in agreement with previous reports. ${ }^{6,7}$ Two typical examples of wheelchair propulsion styles are shown in Figure 2. The recovery phase (time from hand release the hand contact with handrim) in both subjects was considerably longer than push phase (the period between hand contact and hand release from handrim).

There was no significant correlation between push time and the race time (Figure 3). Figure 4 shows that cycle time did not influence the race time. There was also no significant relationship between total push time and the race time (Figure 5). Furthermore, angular velocity did not correlate with the race time (Figure 6). Interestingly, the total push angle correlated significantly with the race time $(P<0.01)$ (Figure 7$)$.

\section{Discussion}

The major finding of the present study was the strong linear correlation between total push angle and the race time in wheelchair marathon racers.

Results of previous studies that examined arm movements during wheelchair propulsion have shown a shorter push time at high wheelchair velocity than at low wheelchair velocity. $3,5,7,8$ The fast wheelchair 
velocity means the short race time. However, the push time in the present study did not correlate with the race time, since our subjects propelled their wheelchairs on the roller using their own optimal velocities. Furthermore, the pattern of arm movement and the push angle were different among subjects in the present study. Previous studies showed that during a high wheelchair velocity, subjects shifted the start and end angles to the front of the hand rim without changing the push angle. ${ }^{5}$ In contrast, the push angle was different at the optimal wheelchair velocities in our subjects and the push times recorded during optimal wheelchair velocities did not correlate with the wheelchair velocities and race time.

In addition, calculation of the push time was inappropriate for analysis of effective wheelchair pushing duration, since the frequency of wheelchair propulsion should be considered within the time of wheelchair propulsion. If a subject selects a high frequency of propulsion using a short push time, the actual push time in a minute would be underestimated. Therefore, the total push time should provide a better analysis of the time components of wheelchair propulsion compared with a simple push time. Our results showed that the total push time did not correlate with the race time in the present study. This finding suggests that time components should not be used to estimate the skill of wheelchair propulsion in long distance racers.

In order to propel the wheelchair, the velocity of the hand pushing the rim of the wheel should be faster than the velocity of the rim. If the former was slower than the latter, the wheelchair should decelerate during the contact of hand with the rim. In the present study, we used the angular velocity to analyze the velocity of pushing handrim, since subjects used handrim of different diameters ${ }^{9}$ and the diameter of the wheels was similar in all wheelchairs. Veeger et $a l^{5}$ suggested that the angular velocity of pushing handrim was the most important factor that determines the speed of propulsion of the wheelchair. However, our results showed a lack of significant relationship between the angular velocity and race time. In fact, while a fast wheelchair speed must be an important factor in a wheelchair long distance race, we suggest that advanced endurance performance is more important than the temporal wheelchair speed. Our subjects had to maintain a continuous high wheelchair speed during $5 \mathrm{~min}$ and their arm movements should improve the endurance performance rather than simply increase of wheelchair velocities. This argument is based on our results that the angular velocity did not correlate with the race time.

A decrease in cycle time resulted in increased cycle frequency and increased velocity of the wheelchair is associated with a decrease in cycle time. ${ }^{3}$ However, cycle time did not correlate with the race time in the present study. The cycle time of wheelchair propulsion is composed of a pushing component and a recovery component. $^{2,3}$ At high wheelchair velocities, there is a decrease in cycle time due to a decrease in push time while the recovery time remains almost constant. ${ }^{3,5,10}$ Our results showed no relationship between push time and the race time, therefore, the cycle time did not correlate with the race time.

The force driving a wheelchair is not determined during the recovery phase, instead, acceleration of the wheelchair occurs during the push phase. The basic mechanism involved in wheelchair propulsion is a direct transformation of the arm force into wheelchair propulsion. We speculated that the optimal force pushing the handrim is dependent on the pushing velocity and duration of pushing. The product of these two parameters is represented by the total push angle. Our results demonstrated a significant relationship between total push angle and the race time. These findings suggest that improvement of either the pushing velocity or pushing duration should shorten the race time.

While neither the total push time nor angular velocity correlated with the race time, the total push angle correlated significantly with the race time in the present study. Angular velocity is calculated by dividing the push angle by push time, thus a short push time is associated with a high angular velocity while a long push time results in a low angular velocity. Thus, the relationship between total push time and angular velocity should be contradictive and reciprocal association. To increase the total push angle, both the total push time and angular velocity have to be improved in a wheelchair long distance race. Since the total push angle represents the total propelling angle per minute, it might be very difficult for a racer to achieve this performance.

The present study investigated the correlation between marathon race times of nine wheelchair athletes and the kinematics of their pushing movement on the hand rim as measured in a biomechanics laboratory. Only one of the variables, total push angle, turned out to be significantly related to race times.

\section{Acknowledgements}

We thank Dr Hatada for his clinical assistance. We are also grateful to Messers Ibusuki, Yamamoto and Takahashi for their physiotherapeutic support. The authors acknowledge the skilful assistance of $\mathrm{Ms}$ Aoki and $\mathrm{Mr}$ Takeda. We also thank Dr FG Issa, from the Department of Medicine, University of Sydney, Sydney, Australia, for the careful reading and editing of the manuscript.

\section{References}

1 Ogata H. A review of wheelchair marathon and tennis. Sangyo Ika Daigaku Zasshi 1994; 16: $201-217$.

2 van-der-Woude LH, Veeger HE, Rozendal RH. Propulsion technique in hand rim wheelchair ambulation. J Med Eng Technol 1989; 13: $136-141$. 
3 Vanlandewijck YC, Spaepen AJ, Lysens RJ. Wheelchair propulsion efficiency: movement pattern adaptations to speed changes. Med Sci Sports Exerc 1994; 26: $1373-1381$.

4 Vanlandewijck YC, Spaepen AJ, Lysens RJ. Wheelchair propulsion: functional ability dependent factors in wheelchair basketball players. Scand J Rehabil Med 1994; 26: 37-48.

5 Veeger HE, van-der-Woude LH, Rozendal RH. Wheelchair propulsion technique at different speeds. Scand J Rehabil Med 1989; 21: $197-203$.

6 Bernard PL et al. Balance and stabilization capability of paraplegic wheelchair athletes. J Rehabil Res Dev 1994; 31: $287-296$.

7 Veeger HE, van-der-Woude LH, Rozendal RH. Effect of handrim velocity on mechanical efficiency in wheelchair propulsion. Med Sci Sports Exerc 1992; 24: 100-107.
8 van-der-Woude LH et al. Wheelchair racing: effects of rim diameter and speed on physiology and technique. Med Sci Sports Exerc 1988; 20: $492-500$.

9 van-der-Linden ML, Valent L, Veeger HE, van-der-Woude LH The effect of wheelchair handrim tube diameter on propulsion efficiency and force application (tube diameter and efficiency in wheelchairs). Ieee Trans Rehabil Eng 1996; 4: 123-132.

10 van-der-Woude LH, Veeger HE, Rozendal RH, Sargeant AJ. Optimum cycle frequencies in hand-rim wheelchair propulsion. Wheelchair propulsion technique. Eur J Appl Physiol 1989; 58: $625-632$. 